\title{
Europäischer Startschuss für die Datenschutzreform: Eine Chance für wirksame Verbesserungen
}

Am 25. Januar 2012 hat die Europäische Kommission das mit großen Erwartungen verknüpfte Reformpaket für den EU-Datenschutz vorgestellt. Das Paket beinhaltet neben einem Vorschlag für eine Verordnung, welche die Datenschutzrichtlinie 95/46/EG aus dem Jahre 1995 ersetzten soll, auch den Entwurf einer Richtlinie für die Datenverarbeitung durch Polizei- und Justizbehörden. Mit dem Reformpaket soll der Datenschutz auf die Höhe der technologischen Entwicklung gebracht und Unterschiede in der nationalen Datenschutzgesetzgebung und Rechtsanwendung in der EU überwunden werden.

Wie bereits mit der Richtlinie von 1995 verfolgt die Kommission auch mit der Verordnung nicht nur rechtliche, sondern auch ökonomische, Ziele. Die Stärkung des von der EU-GrundrechteCharta gewährleisteten Grundrechts auf Schutz personenbezogener Daten ist daher nur eine Seite der Reform. Die andere ist die Stärkung des digitalen Binnenmarktes, der nach Auffassung der Vizepräsidentin und für den Datenschutz zuständigen Kommissarin für Justiz und Bürgerrechte, Viviane Reding, ein größeres Vertrauen der Bürgerinnen und Bürger in Onlinedienste erfordert. Ob der neue Rechtsrahmen dies leisten kann, bleibt abzuwarten, da die Entwürfe noch vom Europäischen Parlament und vom Rat verhandelt werden müssen. Schon während der letzten - kommissionsinternen - Abstimmungen des Reformpakets ist deutlich geworden, mit welcher Energie die verschiedenen Interessengruppen versuchen, den Inhalt in ihrem Sinne zu beeinflussen. Erst am Ende eines vermutlich langwierigen Gesetzgebungsprozesses wird klar sein, ob der Datenschutz insgesamt gewonnen hat und auf einem höheren Niveau in den Mitgliedstaaten der Union angekommen ist.

Ich meine, dass die Kommission hier im Grundsatz auf einem guten Weg ist. Ich begrüße vor allem den neuen Ansatz, dass das EU-Datenschutzrecht künftig auch dann gelten soll, wenn sich Unternehmen aus Nicht-EU-Ländern mit ihren Produkten oder Diensten an Bürger in der EU wenden oder deren Verhalten beobachten. Damit wird eine Lücke im bestehenden Datenschutzrecht geschlossen. Denn die Betreiber von Online-Diensten und sozialen Netzwerken konnten bislang einwenden, dass das EURecht für sie nur gelte, wenn sie über eine Niederlassung innerhalb der EU verfügen.

Für sinnvoll halte ich es auch, dass die Verantwortlichkeit der Daten verarbeitenden Stellen gestärkt werden soll, etwa indem sie ihre internen Strukturen und Abläufe datenschutzgerecht gestalten und dies im Streitfall auch nachgewiesen werden muss. Dieser von den Datenschutzbehörden seit langem geforderte Rechenschaftsgrundsatz (Accountability) wird dazu führen, dass sich Unternehmen weit stärker als bislang mit dem Datenschutz beschäftigen müssen. Flankiert wird dies durch eine Verpflichtung zur Verwendung datenschutzfreundlicher Technologien (Privacy by Design) und zur Vornahme datenschutzfreundlichen Grundeinstellungen (Privacy by Default). Zudem müssen Verarbeitungsvorgänge nachprüfbar dokumentiert und die Folgen von Verarbeitungen, die konkrete Risiken für die Rechte und Freiheiten betroffener Personen bergen, abgeschätzt werden (Privacy Impact Assessment).

Angesichts der guten Erfahrungen in Deutschland unterstütze ich auch, dass die Kommission die Bestellung der betrieblichen und behördlichen Datenschutzbeauftragten europaweit verbindlich machen will. Der Verordnungsvorschlag sieht jedoch vor, dass dies nur für Unternehmen gilt, die mehr als 250 Mitarbeiter beschäftigen. Für Deutschland hieße das, dass nur 0,3 Prozent der inländischen Unternehmen zur Bestellung eines Datenschutzbeauftragten verpflichtet würden. Ich trete daher für eine deutlich niedrigere Grenze ein.

Unterstützenswert sind die Vorstellungen der Kommission auch im Hinblick auf die Stärkung der Datenschutzbehörden in den Mitgliedstaaten. Sie müssen ihre Aufgaben in völliger Unabhängigkeit wahrnehmen und über wirksame Instrumente zur Durchsetzung der Datenschutzanforderungen verfügen. Sie sollen künftig EU-weit über einheitliche Sanktionsmöglichkeiten einschließlich der Verhängung spürbarer Bußgelder - bis zu 2 Prozent des weltweiten Jahresumsatzes - verfügen.

Um die Entscheidungspraxis der Aufsichtsbehörden innerhalb der EU zu harmonisieren, soll eine Pflicht zur Kooperation und gegenseitigen Amtshilfe eingeführt werden. Fälle, die mehr als einen Mitgliedstaat betreffen, sollen einem „Kohärenzverfahren" unterliegen und einer einheitlichen Bewertung durch den Europäischen Datenschutzausschuss - dem Nachfolgegremium der Artikel-29-Gruppe - unterliegen. Ein solches Verfahren ist sinnvoll und notwendig. Zur Wahrung der Unabhängigkeit des Datenschutzes muss die Verfahrensherrschaft allerdings in den Händen der Datenschutzbehörden verbleiben. Damit wäre es nicht vereinbar, wenn die Kommission sich - wie geplant - vorbehält, aufsichtsbehördliche Maßnahmen aufzuhalten oder sogar einzelfallbezogene Beschlüsse zu treffen, wenn sie der Ansicht ist, dass eine Datenschutzbehörde die EU-Verordnung nicht richtig anwendet.

Die Kommission hat mit ihren Vorschlägen insgesamt eine solide Basis für eine Verbesserung des Datenschutzes in der EU gelegt. Parlament und Rat sind nun aufgefordert, hierauf aufzubauen.

Peter Schaar

Der Bundesbeauftragte für den Datenschutz und die Informationsfreiheit 